MATHEMATICS OF COMPUTATION

Volume 70 , Number 233 , Pages 251-267

S 0025-5718(00)01272-2

Article electronically published on June 12, 2000

\title{
ERROR ESTIMATES IN THE NUMERICAL EVALUATION OF SOME BEM SINGULAR INTEGRALS
}

\author{
G. MASTROIANNI AND G. MONEGATO
}

\begin{abstract}
In some applications of Galerkin boundary element methods one has to compute integrals which, after proper normalization, are of the form

$$
\int_{a}^{b} \int_{-1}^{1} \frac{f(x, y)}{x-y} d x d y
$$

where $(a, b) \equiv(-1,1)$, or $(a, b) \equiv(a,-1)$, or $(a, b) \equiv(1, b)$, and $f(x, y)$ is a smooth function.

In this paper we derive error estimates for a numerical approach recently proposed to evaluate the above integral when a $p-$, or $h-p$, formulation of a Galerkin method is used. This approach suggests approximating the inner integral by a quadrature formula of interpolatory type that exactly integrates the Cauchy kernel, and the outer integral by a rule which takes into account the log endpoint singularities of its integrand. Some numerical examples are also given.
\end{abstract}

\section{INTRODUCTION}

In the applications of Galerkin boundary element methods, for the numerical solution of one-dimensional singular and hypersingular integral equations, one has to deal (see [1]) with integrals which after proper normalization are of the form

$$
\int_{a}^{b} \int_{-1}^{1} \frac{f(x, y)}{x-y} d x d y
$$

where $(a, b) \equiv(-1,1)$, or $(a, b) \equiv(a,-1)$, or $(a, b) \equiv(1, b)$, and $f(x, y)$ is a smooth function of both variables. When $y \in(-1,1)$, the inner integral is defined in the Cauchy principal value sense and will be denoted by the symbol $\oint$. In particular, one has to approximate (1.1) by quadrature rules.

Incidentally we note that two-dimensional singular integrals of type (1.1) arise also in the calculation of the aerodynamic load on a lifting body (see 2]), and in this context some numerical procedures for their evaluation have been proposed in [15], 18.

A recent new numerical approach (see [1, 7]) suggests approximating the inner integral by a quadrature formula of interpolatory type, based on the zeros of Legendre polynomials, which exactly integrates the Cauchy kernel times an arbitrary

Received by the editor February 17, 1999.

2000 Mathematics Subject Classification. Primary 41A55; Secondary 65D32, 65N38.

Key words and phrases. Singular integrals, error estimates, boundary element methods.

Work supported by the Consiglio Nazionale delle Ricerche - Comitato Nazionale per le Ricerche Tecnologiche e l'Innovazione, under contract n.96.01875.CT11.

(C)2000 American Mathematical Society 
polynomial of degree $n-1$ and assumes the form

$$
\oint_{-1}^{1} \frac{f(x, y)}{x-y} d x=\sum_{i=1}^{n} w_{n, i}(y) f\left(x_{n, i}, y\right)+R_{n}^{I}(f ; y),
$$

whenever $y \in(-1,1)$ or $y \notin(-1,1)$ but is very close to one of the endpoints. For the construction of these rules see [12. When $y$ is sufficiently away from $(-1,1)$, then the integral is evaluated by a standard Gauss-Legendre formula. The outer integral should be computed using rules like those proposed in [14, Remark 1] or [16, which take into account the endpoint singular behaviour of the integrand function. These rules are of the form

$$
\int_{a}^{b} F(y) d y=\sum_{j=1}^{m} v_{m, j} F\left(y_{m, j}\right)+R_{m}(F),
$$

with $v_{m, j}>0, j=1, \ldots, m$, and, taking for example $(a, b) \equiv(-1,1)$, they satisfy the convergence property

$$
\lim _{m \rightarrow \infty} \sum_{j=1}^{m} v_{m, j} \frac{\log ^{k}\left(1-y_{m, j}^{2}\right)}{\left(1-y_{m, j}^{2}\right)^{\beta}}=\int_{-1}^{1} \frac{\log ^{k}\left(1-y^{2}\right)}{\left(1-y^{2}\right)^{\beta}} d y
$$

for $\beta<1, k=0,1$. More generally, they converge whenever $F(y)$ has only integrable endpoint singularities.

The composition of (1.2) and (1.3) then leads to the final formula

$$
\int_{a}^{b} \int_{-1}^{1} \frac{f(x, y)}{x-y} d x d y=\sum_{j=1}^{m} v_{m, j} \sum_{i=1}^{n} w_{n, i}\left(y_{m, j}\right) f\left(x_{n, i}, y_{m, j}\right)+R_{m, n}(f),
$$

where

$$
R_{m, n}(f)=\sum_{j=1}^{m} v_{m, j} R_{n}^{I}\left(f ; y_{m, j}\right)+R_{m}\left(\int_{-1}^{1} \frac{f(x, \cdot)}{x-\cdot} d x\right) .
$$

Following [1], here we are referring to a $p$-formulation of the Galerkin BEM and are interested in the accurate calculation of the corresponding integrals of form (1.1). Therefore, we are not concerned with the behaviour of the quadrature rule, having fixed the number of its nodes, as the size of the domain of integration tends to zero. Instead, given a boundary element of fixed length, we let the number of points tend to infinity and want to know the behaviour of $R_{m, n}(f)$.

Since estimates for $R_{m}$ are either available or easy to obtain (see [14, 16] and Section 3), to determine the behaviour of $R_{m, n}(f)$, as $m, n \rightarrow \infty$, we need to have accurate pointwise (with respect to $y$ ) bounds for $R_{n}^{I}(f ; y)$. All known estimates refer to a variable $y$ bounded away from the endpoints \pm 1 (see for example [3], [12]). In this paper we derive for (1.2) a pointwise error estimate which, taking into account property (1.4), will then allow us to obtain a bound for the global term (1.6).

The estimate we derive for $R_{n}^{I}(f ; y)$ may be useful for other types of applications. For example it could be used to obtain uniform (with respect to the collocation point) error estimates for the evaluation of integrals required by a collocation method, or to obtain weighted uniform estimates for Nyström-type interpolants for second kind singular integral equations on bounded intervals. 


\section{Pointwise ERROR ESTIMATES FOR THE INNER INTEGRAL}

For the sake of generality, we will derive a pointwise error estimate for a quadrature rule of type (1.2) when a symmetric Jacobi weight function $w^{\alpha}(x):=$ $\left(1-x^{2}\right)^{\alpha}, \alpha>-1$, is also present. Our results could be proved for a more general Jacobi weight function $w^{\alpha, \beta}(x)=(1-x)^{\alpha}(1+x)^{\beta}, \alpha, \beta>-1$, but this would introduce more cases to be examined in the proofs, depending upon the values of the two parameters $\alpha, \beta$; the proofs would be very similar anyway.

Thus we consider the quadrature rule

$$
\oint_{-1}^{1} w^{\alpha}(x) \frac{f(x, y)}{x-y} d x=\sum_{i=1}^{n} w_{n, i}(y) f\left(x_{n, i}, y\right)+R_{n}^{I}(f ; y) .
$$

It is of interpolatory type and is obtained by replacing, for any given $y, f(x, y)$ by its Lagrange interpolation (with respect to $x$ ) polynomial of degree $n-1$, that we shall denote by $L_{n}(f, y ; x)$, based on the zeros of the $n$ th-degree Jacobi polynomial $P_{n}^{(\alpha, \alpha)}(x):-1<x_{n, n}<\cdots<x_{n, 1}<1$ (see [19]).

In the following we define

$$
H(f ; y):=\oint_{-1}^{1} w^{\alpha}(x) \frac{f(x, y)}{x-y} d x .
$$

We shall also make use of the quantities (see [8, (12.1.2)])

$$
\begin{gathered}
\Delta_{1, h \phi}^{r} f(x, y):=\sum_{k=0}^{r}(-1)^{k}\left(\begin{array}{l}
r \\
k
\end{array}\right) f\left(x+\left(\frac{r}{2}-k\right) h \phi(x), y\right), \\
\Delta_{2, h \phi}^{r} f(x, y):=\sum_{k=0}^{r}(-1)^{k}\left(\begin{array}{l}
r \\
k
\end{array}\right) f\left(x, y+\left(\frac{r}{2}-k\right) h \phi(y)\right), \\
\omega_{I}^{r}(f, t)=\omega_{I}^{r}(f, t)_{\infty}:=\sup _{0<h \leq t} \max \left\{\left\|\Delta_{1, h \phi}^{r} f\right\|_{I},\left\|\Delta_{2, h \phi}^{r} f\right\|_{I}\right\}, \quad t<1,
\end{gathered}
$$

where $h>0, r \geq 1$ an integer, $\phi(z):=\sqrt{1-z^{2}}, I:=[-1,1]^{2}$,

$$
\|f\|_{\infty} \equiv\|f\|_{I}:=\sup _{I}|f(x, y)|
$$

and

$$
\Delta_{i, h \phi}^{r} f(x, y):=0
$$

whenever one of the arguments of $f$ on the right-hand sides of the above definitions does not belong to $I$.

Incidentally, we notice that if in the definition of $\Delta_{i, h \phi}^{r} f$ we choose $\phi \equiv 1$, then the corresponding $\omega_{I}^{r}$ reduces to the ordinary modulus of continuity, which is greater than or equal to that defined in (2.3). To obtain accurate pointwise error estimates for (2.1), we need to use (2.3). This is because, among other properties, the new modulus satisfies the following two inequalities:

$$
\begin{gathered}
E_{m}(f)_{\infty} \leq \omega_{I}^{r}\left(f, \frac{1}{m}\right) \quad(\text { see }[8,(12.1 .3)]), \\
\omega_{I}^{r}(f, t) \leq c t^{r} \sum_{k=0}^{\lfloor 1 / t\rfloor}(k+1)^{r-1} E_{k}(f)_{\infty} \quad \text { (see [8, (12.1.4)]), }
\end{gathered}
$$


where*

$$
E_{k}(f)_{\infty}:=\inf _{p_{k} \in \Pi_{k}}\left\|f-p_{k}\right\|_{\infty} .
$$

These bounds will be fundamental in deriving our results.

For notational convenience, we shall also set $\omega_{I}(f, t):=\omega_{I}^{1}(f, t)$; moreover, the symbol $c$ will denote a constant which in general will take different values at different occurences.

Theorem 1. Let $\alpha>-1$ and $y \in(-1,1)$. For any $f \in C[-1,1]^{2}$ we have

$$
|H(f ; y)| \leq c h_{\alpha}(y)\left[\|f\|_{\infty}+\int_{0}^{1} \frac{\omega_{I}(f ; u)}{u} d u\right]
$$

with

$$
h_{\alpha}(y)= \begin{cases}w^{\alpha}(y), & -1<\alpha<0, \\ \log \frac{1}{1-y^{2}}, & \alpha=0, \\ 1, & \alpha>0,\end{cases}
$$

where the constant $c=c(\alpha)$ depends only upon $\alpha$.

Proof. Assume first $-1<y<-1 / 2$; then, since $-1<2 y+1<0$ and $2 y+1>y$, we write

$$
\oint_{-1}^{1} w^{\alpha}(x) \frac{f(x, y)}{x-y} d x=\oint_{-1}^{2 y+1}+\int_{2 y+1}^{1}=: I_{1}+I_{2} .
$$

To bound $I_{2}$ we proceed as follows. Notice first that $x \geq 2 y+1$ implies $0<$ $\frac{1+x}{x-y} \leq 2 ;$ therefore we have

$$
\left|I_{2}\right| \leq 2\|f\|_{\infty} \int_{2 y+1}^{1}(1-x)^{\alpha}(1+x)^{\alpha-1} d x .
$$

Moreover,

$$
\int_{2 y+1}^{1}(1-x)^{\alpha}(1+x)^{\alpha-1} d x \leq c\left[\int_{2 y+1}^{0}(1+x)^{\alpha-1} d x+\int_{0}^{1}(1-x)^{\alpha} d x\right] \leq c h_{\alpha}(y) .
$$

By inserting the latter bound into (2.8) we obtain

$$
\left|I_{2}\right| \leq 2 c\|f\|_{\infty}(1+y)^{\alpha} .
$$

To bound $I_{1}$, first we note that

$$
\oint_{-1}^{2 y+1} \frac{d x}{x-y}=0
$$

hence write

$$
\begin{aligned}
I_{1}= & w^{\alpha}(y) \int_{y-(1+y)}^{y+(1+y)} \frac{f(x, y)-f(y, y)}{x-y} d x \\
& +\int_{-1}^{2 y+1} f(x, y) \frac{w^{\alpha}(x)-w^{\alpha}(y)}{x-y} d x=: I_{1}^{\prime}+I_{1}^{\prime \prime} .
\end{aligned}
$$

\footnotetext{
${ }^{*} \Pi_{k}$ below denotes the space of all polynomials of degree $k$ in each variable.
} 
We have

$$
I_{1}^{\prime}=w^{\alpha}(y)\left[\int_{y-(1+y)}^{y} \frac{f(x, y)-f(y, y)}{x-y} d x+\int_{y}^{y+(1+y)} \frac{f(x, y)-f(y, y)}{x-y} d x\right],
$$

hence, setting $x-y=u \phi(y) / 2$,

$$
\begin{aligned}
\left|I_{1}^{\prime}\right| & =w^{\alpha}(y)\left|\int_{0}^{2} \frac{f(y+u \phi(y) / 2, y)-f(y-u \phi(y) / 2, y)}{u} d u\right| \\
& \leq w^{\alpha}(y) \int_{0}^{1} \frac{\left|\Delta_{1, u \phi}^{1} f(y, y)\right|}{u} d u+2 w^{\alpha}(y)\|f\|_{\infty} .
\end{aligned}
$$

Therefore, recalling definition (2.3) we have

$$
\left|I_{1}^{\prime}\right| \leq w^{\alpha}(y) \int_{0}^{1} \frac{\omega_{I}(f, u)}{u} d u+2 w^{\alpha}(y)\|f\|_{\infty} .
$$

To bound $I_{1}^{\prime \prime}$ we notice first that

$$
\frac{w^{\alpha}(x)-w^{\alpha}(y)}{x-y}=(1+x)^{\alpha}\left[\frac{(1-x)^{\alpha}-(1-y)^{\alpha}}{x-y}\right]+(1-y)^{\alpha}\left[\frac{(1+x)^{\alpha}-(1+y)^{\alpha}}{x-y}\right],
$$

and then write

$$
\begin{aligned}
\left|I_{1}^{\prime \prime}\right| \leq & \|f\|_{\infty}\left[\int_{-1}^{2 y+1}(1+x)^{\alpha}\left|\frac{(1-x)^{\alpha}-(1-y)^{\alpha}}{x-y}\right| d x\right. \\
& \left.+(1-y)^{\alpha} \int_{-1}^{2 y+1}\left|\frac{(1+x)^{\alpha}-(1+y)^{\alpha}}{x-y}\right| d x\right] .
\end{aligned}
$$

Since $y<-1 / 2$ and $x \leq 2 y+1<0$, we have

$$
\left|\frac{(1-x)^{\alpha}-(1-y)^{\alpha}}{x-y}\right| \leq c .
$$

Moreover, setting $1+x=u(1+y)$ we obtain

$$
\frac{(1+x)^{\alpha}-(1+y)^{\alpha}}{x-y}=(1+y)^{\alpha-1} \frac{u^{\alpha}-1}{u-1} .
$$

Thus, from (2.11) we have

$$
\left|I_{1}^{\prime \prime}\right| \leq c\|f\|_{\infty}\left[(1+y)^{\alpha+1}+w^{\alpha}(y) \int_{0}^{2}\left|\frac{u^{\alpha}-1}{u-1}\right| d u\right] \leq c\|f\|_{\infty} w^{\alpha}(y) .
$$

Combining (2.9), (2.10) and (2.12), we finally obtain

$$
\left|\oint_{-1}^{1} w^{\alpha}(x) \frac{f(x, y)}{x-y} d x\right| \leq c w^{\alpha}(y)\left[\|f\|_{\infty}+\int_{0}^{1} \frac{\omega_{I}(f, u)}{u} d u\right],
$$

where $c=c(\alpha)$ depends only upon $\alpha$.

The cases $\alpha=0$ and $\alpha>0$ are even simpler. They can be easily derived by repeating the preceding arguments, after having introduced some obvious simplifications. The symmetric situation $1 / 2<y<1$ is quite similar; indeed it is sufficient to replace $y$ by $-y$ in the proof above.

The final case $-1 / 2 \leq y \leq 1 / 2$ is fairly simple and can be dealt with as follows. Let us consider

$$
A=\oint_{-1}^{1} w^{\alpha}(x) \frac{f(x, y)}{x-y} d x=\int_{-1}^{y-\frac{1}{4}}+\oint_{y-\frac{1}{4}}^{y+\frac{1}{4}}+\int_{y+\frac{1}{4}}^{1}=: A_{1}+A_{2}+A_{3} .
$$


We have

$$
\left|A_{1}\right| \leq c\|f\|_{\infty} \int_{-1}^{y-\frac{1}{4}} w^{\alpha}(x) d x \leq c\|f\|_{\infty}
$$

and, similarly,

$$
\left|A_{3}\right| \leq c\|f\|_{\infty}
$$

For the remaining integral we proceed as follows. Write first

$$
A_{2}=w^{\alpha}(y) \int_{y-\frac{1}{4}}^{y+\frac{1}{4}} \frac{f(x, y)-f(y, y)}{x-y} d x+\int_{y-\frac{1}{4}}^{y+\frac{1}{4}} \frac{w^{\alpha}(x)-w^{\alpha}(y)}{x-y} d x .
$$

Hence, setting $x=y+u \phi(y) / 2$, note that

$$
\begin{aligned}
& \left|\int_{y-\frac{1}{4}}^{y+\frac{1}{4}} \frac{f(x, y)-f(y, y)}{x-y} d x\right|=\left|\int_{-\frac{1}{2 \phi(y)}}^{\frac{1}{2 \phi(y)}} \frac{f(y+u \phi(y) / 2, y)-f(y, y)}{u} d u\right| \\
& \leq c \int_{0}^{\frac{1}{2 \phi(y)}} \frac{|f(y+u \phi(y) / 2, y)-f(y-u \phi(y) / 2, y)|}{u} d u \leq c \int_{0}^{1} \frac{\omega_{I}(f, u)}{u} d u .
\end{aligned}
$$

By inserting this bound into (2.15) we obtain

$$
\left|A_{2}\right| \leq c\left[\|f\|_{\infty}+\int_{0}^{1} \frac{\omega_{I}(f, u)}{u} d u\right]
$$

and finally, from (2.14),

$$
|A| \leq c w^{\alpha}(y)\left[\|f\|_{\infty}+\int_{0}^{1} \frac{\omega_{I}(f, u)}{u} d u\right],
$$

i.e., (2.7).

Theorem 2. Given any polynomial $p_{m}(x, y)$ of degree $m>1$ in $x$ and $y$, and a function $f(x, y)$ such that

$$
\int_{0}^{1} \frac{\omega_{I}(f, u)}{u} d u<\infty
$$

we have

$$
\left|H\left(f-p_{m} ; y\right)\right| \leq c h_{\alpha}(y)\left[\left\|f-p_{m}\right\|_{\infty} \log m+\int_{0}^{\frac{1}{m}} \frac{\omega_{I}^{r}(f, u)}{u} d u\right],-1<y<1,
$$

for any $\alpha>-1$ and $r \geq 1$, where the constant $c=c(\alpha)$ depends only upon $\alpha$ and $h_{\alpha}(y)$ is defined as in Theorem 1.

Proof. Recalling (2.4), we consider first the term

$$
\int_{0}^{1} \frac{\omega_{I}\left(f-p_{m}, u\right)}{u} d u=\int_{0}^{\frac{1}{m}}+\int_{\frac{1}{m}}^{1}=: B_{1}+B_{2},
$$

and note that

$$
\left|B_{2}\right| \leq c\left\|f-p_{m}\right\|_{\infty} \log m, m>1,
$$

since from the definition of $\omega_{I}$ it follows that

$$
\omega_{I}(f, u) \leq c\|f\|_{\infty} .
$$


To bound $B_{1}$ we use inequality (2.5) with $r=1$ and

$$
E_{k}\left(f-p_{m}\right)_{\infty} \leq \begin{cases}\left\|f-p_{m}\right\|_{\infty}, & k \leq m, \\ E_{k}(f)_{\infty}, & k>m .\end{cases}
$$

The bound (2.18) follows directly from the definition of $E_{k}(f)_{\infty}$. These inequalities allow us to write

$$
\omega_{I}\left(f-p_{m}, u\right) \leq c u \sum_{k=0}^{\lfloor 1 / u\rfloor} E_{k}\left(f-p_{m}\right)_{\infty} \leq c u\left[(m+1)\left\|f-p_{m}\right\|_{\infty}+\sum_{k \geq m+1}^{\lfloor 1 / u\rfloor} E_{k}(f)_{\infty} .\right.
$$

Recalling (2.4) we further have

$$
\begin{gathered}
\sum_{k=m+1}^{\lfloor 1 / u\rfloor} E_{k}(f)_{\infty} \leq c \sum_{k=m}^{\lfloor 1 / u\rfloor-1} \omega_{I}^{r}\left(f, \frac{1}{k+1}\right) \\
\leq c \sum_{k=m}^{\lfloor 1 / u\rfloor-1} \int_{k}^{k+1} \omega_{I}^{r}\left(f, \frac{1}{v}\right) d v \leq c \int_{m}^{\frac{1}{u}} \omega_{I}^{r}\left(f ; \frac{1}{v}\right) d v=c \int_{u}^{\frac{1}{m}} \frac{\omega_{I}^{r}(f, t)}{t^{2}} d t, r \geq 1 .
\end{gathered}
$$

Thus (2.19) becomes

$$
\omega_{I}\left(f-p_{m}, u\right) \leq c u\left[(m+1)\left\|f-p_{m}\right\|_{\infty}+\int_{u}^{\frac{1}{m}} \frac{\omega_{I}^{r}(f, t)}{t^{2}} d t\right], r \geq 1,
$$

and the integral $B_{1}$ can be estimated as

$$
\left|B_{1}\right| \leq c\left[\left\|f-p_{m}\right\|_{\infty}+\int_{0}^{\frac{1}{m}} \int_{u}^{\frac{1}{m}} \frac{\omega_{I}^{r}(f, t)}{t^{2}} d t d u\right], r \geq 1 .
$$

By interchanging the order of integration, the last double integral can be rewritten as

$$
\int_{0}^{\frac{1}{m}} \frac{\omega_{I}^{r}(f, t)}{t} d t
$$

Finally, from (2.22) and (2.17) we derive the bound

$$
\int_{0}^{1} \frac{\omega_{I}\left(f-p_{m} ; u\right)}{u} d u \leq c\left[\left\|f-p_{m}\right\|_{\infty} \log m+\int_{0}^{\frac{1}{m}} \frac{\omega_{I}^{r}(f ; u)}{u} d u\right] .
$$

Combining the latter with Theorem 1, we obtain (2.16).

Remark 1. If $\omega_{I}^{r}(f, u)=O\left(u^{\gamma}\right)$ for some real $\gamma>0$, then

$$
\int_{0}^{\frac{1}{m}} \frac{\omega_{I}^{r}(f, u)}{u} d u=O\left(m^{-\gamma}\right) .
$$

In particular, when $f \in H_{p}(\mu, \mu)$, where $H_{p}(\mu, \mu)$ denotes the space of functions with all partial derivatives of order $\leq p$ continuous on $[-1,1]^{2}$, and with those of order $p$ that are Hölder continuous of degree $\mu$, we have

$$
\omega_{I}^{r}(f, u)=O\left(u^{p+\mu}\right),
$$

for any integer $r \geq p+\mu$, hence

$$
\int_{0}^{\frac{1}{m}} \frac{\omega_{I}^{r}(f, u)}{u} d u=O\left(m^{-p-\mu}\right) .
$$


Theorem 3. Let $-\frac{1}{2} \leq \alpha \leq \frac{1}{2}$ and $y \in(-1,1)$. Given any $f \in C[-1,1]^{2}$, for the remainder term in (2.1) we have

$$
\left|R_{n}^{I}(f ; y)\right| \leq c \bar{h}_{\alpha}(y)\left[E_{n-1}(f)_{\infty} \log n+\int_{0}^{\frac{1}{n-1}} \frac{\omega_{I}^{r}(f, u)}{u} d u\right], \quad n>1,
$$

where

$$
\bar{h}_{\alpha}(y)= \begin{cases}w^{\frac{\alpha}{2}-\frac{1}{4}}(y), & \alpha<0, \\ w^{-\frac{1}{4}}(y) \log \frac{1}{1-y^{2}}, & \alpha=0, \\ w^{-\frac{1}{4}}(y), & \alpha>0,\end{cases}
$$

and $c=c(\alpha)$ depends only upon $\alpha$.

Proof. From the representation

$$
R_{n}^{I}(f ; y)=\int_{-1}^{1} w^{\alpha}(x) \frac{f(x, y)-L_{n}(f, y ; x)}{x-y} d x,
$$

we know that the bound

$$
\begin{aligned}
\left|R_{n}^{I}(f ; y)\right| \leq & \left|\int_{-1}^{1} w^{\alpha}(x) \frac{f(x, y)-p_{m}(x, y)}{x-y} d x\right| \\
& +\left|\int_{-1}^{1} w^{\alpha}(x) \frac{L_{n}\left(f-p_{m}, y ; x\right)}{x-y} d x\right|=: I_{1}+I_{2}
\end{aligned}
$$

holds for any polynomial $p_{m}$ of degree $m \leq n-1$ with respect to each variable. By taking as $p_{m}$ a "best" (uniform) approximation polynomial of degree $n-1$ defined by (2.18), from Theorem 2 we immediately derive

$$
\left|I_{1}\right| \leq c h_{\alpha}(y)\left[E_{n-1}(f)_{\infty} \log n+\int_{0}^{\frac{1}{n-1}} \frac{\omega_{I}^{r}(f, u)}{u} d u\right] .
$$

To bound $I_{2}$, for notational convenience we set $e_{m}=f-p_{m}$ and write

$$
I_{2}=\int_{-1}^{1} w^{\alpha}(x) \frac{L_{n}\left(e_{m}, y ; x\right)-L_{n}\left(e_{m}, y ; y\right)}{x-y} d x+L_{n}\left(e_{m}, y ; y\right) \int_{-1}^{1} \frac{w^{\alpha}(x)}{x-y} d x .
$$

Then we discretize the first integral by means of the corresponding $n$-point Gaussian rule with nodes $\left\{x_{n, i}\right\}$ and weights $\left\{\lambda_{n, i}\right\}$; we obtain

$$
I_{2}=\sum_{i=1}^{n} \lambda_{n, i} \frac{L_{n}\left(e_{m}, y ; x_{n, i}\right)-L_{n}\left(e_{m}, y ; y\right)}{x_{n, i}-y}+L_{n}\left(e_{m}, y ; y\right) \int_{-1}^{1} \frac{w^{\alpha}(x)}{x-y} d x
$$

if all $x_{n, i} \neq y$, or

$$
\begin{gathered}
I_{2}=\sum_{i=1, i \neq j}^{n} \lambda_{n, i} \frac{L_{n}\left(e_{m}, y ; x_{n, i}\right)-L_{n}\left(e_{m}, y ; y\right)}{x_{n, i}-y}+\lambda_{n, j} L_{n}^{\prime}\left(e_{m}, y ; y\right) \\
+L_{n}\left(e_{m}, y ; y\right) \int_{-1}^{1} \frac{w^{\alpha}(x)}{x-y} d x
\end{gathered}
$$

if $x_{n, j}=y$. 
For simplicity we examine only (2.26), since the case of (2.27) is very similar. Expression (2.26) can be reformulated as

$$
\begin{aligned}
I_{2}= & A^{*}(y) L_{n}\left(e_{m}, y ; y\right) \\
& +\sum_{i=1, i \neq i_{c}}^{n} \lambda_{n, i} \frac{e_{m}\left(x_{n, i} ; y\right)}{x_{n, i}-y}+\lambda_{n, i_{c}} \frac{L_{n}\left(e_{m}, y ; x_{n, i_{c}}\right)-L_{n}\left(e_{m}, y ; y\right)}{x_{n, i_{c}}-y} \\
= & : A_{1}+A_{2}+A_{3},
\end{aligned}
$$

where

$$
A^{*}(y)=\int_{-1}^{1} \frac{w^{\alpha}(x)}{x-y} d x-\sum_{i=1, i \neq i_{c}}^{n} \frac{\lambda_{n, i}}{x_{n, i}-y}
$$

and $x_{n, i_{c}}$ denotes the closest node to $y$. Notice that since $m=n-1$, above we have used the property $L_{n}\left(e_{m}, y ; x_{n, i}\right)=e_{m}\left(x_{n, i} ; y\right)$.

We recall (see for example [6, Lemma 5.3]) that the following bound has been proved:

$$
\left|A^{*}(y)\right| \leq c h_{\alpha}(y),
$$

where $h_{\alpha}(y)$ is defined as in (2.7). Moreover, since (see [10, (2.7) and (2.11)]) for $\alpha \geq-1 / 2$

$$
\left\|w^{\frac{\alpha}{2}+\frac{1}{4}} L_{n}\left(e_{m}, y ; \cdot\right)\right\|_{\infty} \leq c\left\|e_{m}\right\|_{\infty} \log n,
$$

for $A_{1}$ we have

$$
\left|A_{1}\right| \leq c \frac{h_{\alpha}(y)}{w^{\frac{\alpha}{2}+\frac{1}{4}}(y)}\left\|e_{m}\right\|_{\infty} \log n .
$$

To bound $A_{2}$ we recall preliminarily that from [4, Lemma 3.4] we have

$$
\sum_{i=1, i \neq i_{c}}^{n} \frac{\lambda_{n, i}}{\left|x_{n, i}-y\right|} \leq c h_{\alpha}(y) \log n,
$$

hence

$$
\left|A_{2}\right| \leq c h_{\alpha}(y)\left\|e_{m}\right\|_{\infty} \log n .
$$

In the case of $A_{3}$ we rewrite the corresponding expression in the form

$$
A_{3}=\lambda_{n, i_{c}} L_{n}^{\prime}\left(e_{m}, y ; \xi_{n, i_{c}}\right), \quad\left|y-\xi_{n, i_{c}}\right|<\left|y-x_{n, i_{c}}\right|,
$$

recall (see [17, p.370]) the estimate

$$
\lambda_{n, i_{c}} \sim \frac{\sqrt{1-x_{n, i_{c}}^{2}}}{n} w^{\alpha}\left(x_{n, i_{c}}\right),
$$

and note that for $y \in[-1+\delta, 1-\delta], \delta=\left(1-x_{n, 1}\right) / 2$, we have

$$
1 \pm \xi_{n, i_{c}} \sim 1 \pm x_{n, i_{c}} \sim 1 \pm y,
$$

where $a \sim b$ means that there exist two positive constants $c_{1}, c_{2}$ such that $c_{1} \leq$ $a / b \leq c_{2}$. We obtain

$$
\left|A_{3}\right| \leq \frac{c}{n} w^{\alpha+1 / 2}\left(\xi_{n, i_{c}}\right)\left|L_{n}^{\prime}\left(e_{m}, y ; \xi_{n, i_{c}}\right)\right|
$$

hence

$$
\left|A_{3}\right| \leq c w^{\frac{\alpha}{2}-\frac{1}{4}}\left(\xi_{n, i_{c}}\right)\left\|w^{\frac{\alpha}{2}+\frac{1}{4}} L_{n}\left(e_{m}, y ; \cdot\right)\right\|_{\infty} \leq c w^{\frac{\alpha}{2}-\frac{1}{4}}(y)\left\|e_{m}\right\|_{\infty} \log n,
$$


since

$$
\frac{1}{k}\left\|w^{\beta+1 / 2} p_{k}^{\prime}\right\|_{\infty} \leq c\left\|w^{\beta} p_{k}\right\|_{\infty}, \quad \beta \geq 0,
$$

for any polynomial of degree $k \geq 1$. This last estimate follows almost immediately from [17, Lemma 2] (see, for example, 11, Corollary 2.3]). Finally,

$$
\left|A_{3}\right| \leq c w^{\frac{\alpha}{2}-\frac{1}{4}}(y)\left\|e_{m}\right\|_{\infty} \log m .
$$

From (2.28), (2.29) and (2.31) bound (2.23) follows.

The case $y \notin[-1+\delta, 1-\delta]$ follows directly from expression (2.26) and the above bounds once we rewrite it in the form

$$
I_{2}=\left[A_{2}+\frac{\lambda_{n, i_{c}}}{x_{n, i_{c}}-y} e_{m}\left(x_{n, i_{c}} ; y\right)\right]+L_{n}\left(e_{m} ; y\right)\left[A^{*}(y)+\frac{\lambda_{n, i_{c}}}{x_{n, i_{c}}-y}\right],
$$

where $i_{c}$ is either 1 or $n$. To this end we further need to recall the symmetry of the nodes $x_{n, i}$, hence notice that from (2.30), the estimate (see [19]) $1-x_{n, 1} \sim n^{-2}$ and the bound $y-x_{n, 1}>\delta$ we also have

$$
\frac{\lambda_{n, 1}}{x_{n, 1}-y} \leq c w^{\alpha}\left(x_{n, 1}\right) \leq c \begin{cases}1 & \text { if } \alpha \geq 0 \\ w^{\alpha}(y) & \text { if } \alpha<0\end{cases}
$$

Remark 2. If in (2.23) $f \in H_{p}(\mu, \mu), 0<\mu \leq 1$, then

$$
\left|E_{n-1}(f)\right| \leq c \int_{0}^{\frac{1}{n-1}} \frac{\omega_{I}^{r}(f ; u)}{u} d u=O\left(n^{-p-\mu}\right)
$$

for any given integer $r \geq p+1$, and

$$
\left|R_{n}^{I}(f ; y)\right| \leq \frac{c \log n}{n^{p+\mu}} \bar{h}_{\alpha}(y) .
$$

Next we examine the situation $|y|>1$. The derivation of the corresponding results is fairly simple.

Lemma 1. For any real $\beta>-1$ and $|y|>1$, we have

$$
\left|\int_{-1}^{1} \frac{w^{\beta}(x)}{x-y} d x\right| \leq c \delta_{\beta}(y)
$$

where

$$
\delta_{\beta}(y)= \begin{cases}1, & \beta>0, \\ \left|\log \left(y^{2}-1\right)\right|, & \beta=0, \\ \left(y^{2}-1\right)^{\beta}, & \beta<0,\end{cases}
$$

and the constant $c=c(\beta)$ depends only upon $\beta$.

Proof. When $\beta=0$ a direct calculation of the above integral gives the bound $\left|\log \left(y^{2}-1\right)\right|$. When $\beta \neq 0$, it is sufficient to consider the integral

$$
I=\int_{0}^{1} \frac{(1-x)^{\beta}}{y-x} d x, y>1 .
$$

For $\beta>0$ this integral can be bounded by

$$
|I| \leq \int_{0}^{1}(1-x)^{\beta-1} d x \leq c .
$$


For $\beta<0$ we could represent $I$ by using the Gauss hypergeometric function ${ }_{2} F_{1}(1,1, \beta+2 ; 1 / y)$ (see [9]); however this becomes singular as $y \rightarrow 1^{+}$and we should derive its behaviour. For our purposes it is sufficient to proceed in the following elementary way.

Write

$$
I=(y-1)^{\beta} \int_{0}^{1} \frac{\left(\frac{1-x}{y-1}\right)^{\beta}}{y-x} d x
$$

and set $t=\frac{1-x}{y-1}$. We obtain, assuming $y<2$,

$$
\begin{aligned}
I & =(y-1)^{\beta} \int_{0}^{\frac{1}{y-1}} \frac{t^{\beta}}{t+1} d t \\
& \leq(y-1)^{\beta}\left[\int_{0}^{1} t^{\beta} d t+\int_{1}^{\frac{1}{y-1}} t^{\beta-1} d t\right] \leq c(y-1)^{\beta} .
\end{aligned}
$$

By considering then the corresponding integral over $(-1,0)$ we finally obtain $(2.33)$.

Since in the present case the results corresponding to those of Theorems 1 and 2 are trivial to derive, we state explicitly only the one concerning the behaviour of the remainder term $R_{n}^{I}(f ; y)$.

Theorem 4. Let $\alpha \geq-1 / 2$ and $|y|>1$. Given any $f \in C[-1,1]^{2}$, for the remainder term in (2.1) we have

$$
\left|R_{n}^{I}(f ; y)\right| \leq c \bar{\delta}_{\alpha}(y) E_{n-1}(f)_{\infty} \log n,
$$

where

$$
\bar{\delta}_{\alpha}(y)= \begin{cases}\left|\log \left(y^{2}-1\right)\right| & \text { if } \alpha=\frac{1}{2}, \\ 1 & \text { if } \alpha>\frac{1}{2}, \\ \left(y^{2}-1\right)^{\frac{\alpha}{2}-\frac{1}{4}} & \text { otherwise. }\end{cases}
$$

Proof. We note preliminarily that

$$
\left|\int_{-1}^{1} w^{\alpha}(x) \frac{f(x, y)}{x-y} d x\right| \leq c\|f\|_{\infty} \int_{-1}^{1} \frac{w^{\alpha}(x)}{|x-y|} d x=c \delta_{\alpha}(y)\|f\|_{\infty},
$$

where $\delta_{\alpha}(y)$ is defined as in Lemma 2. Then we consider

$$
\begin{aligned}
\left|R_{n}^{I}(f ; y)\right| \leq & \left|\int_{-1}^{1} w^{\alpha}(x) \frac{f(x, y)-p_{m}(x, y)}{x-y} d x\right| \\
& +\left|\int_{-1}^{1} w^{\alpha}(x) \frac{L_{n}\left(f-p_{m}, y ; x\right)}{x-y} d x\right|=: I_{1}+I_{2},
\end{aligned}
$$

where $p_{m}$ denotes a best (uniform) approximation polynomial of degree $n-1$ associated with $f$. Recalling Lemma 2 , it is then straightforward to derive for $I_{1}$ the bound

$$
\left|I_{1}\right| \leq c \delta_{\alpha}(y) E_{n-1}(f)_{\infty}
$$


To bound $I_{2}$ we need to assume $\alpha \geq-1 / 2$. In this case we have

$$
\begin{aligned}
\left|I_{2}\right| & =\left|\int_{-1}^{1} w^{\frac{\alpha}{2}-\frac{1}{4}}(x) \frac{w^{\frac{\alpha}{2}+\frac{1}{4}}(x) L_{n}\left(f-p_{m}, y ; x\right)}{x-y} d x\right| \\
& \leq\left\|w^{\frac{\alpha}{2}+\frac{1}{4}} L_{n}\left(f-p_{m}\right)\right\|_{\infty} \int_{-1}^{1} \frac{w^{\frac{\alpha}{2}-\frac{1}{4}}(x)}{|x-y|} \leq c \delta_{\frac{\alpha}{2}-\frac{1}{4}}(y) E_{n-1}(f)_{\infty} \log n .
\end{aligned}
$$

This proves (2.34).

\section{BehaViour of THE GLOBAL ERROR AND NUMERICAL EXAMPLES}

For the remainder term of our quadrature formula (1.2), in Section 2 we have derived a pointwise bound which is, whenever $f \in H_{p}(\mu, \mu), p \geq 0$, of the form

$$
\left|R_{n}^{I}(f ; y)\right| \leq c g(y) n^{-p-\mu} \log n .
$$

where the constant $c$ is independent of $y$ and $n$, while the function $g(y)$ is $\left(1-y^{2}\right)^{-\frac{1}{4}} \log \frac{1}{1-y^{2}}$ when $|y|<1$, and $\left(y^{2}-1\right)^{-\frac{1}{4}}$ when $|y|>1$.

The quadratures we suggest using to evaluate the outer integral have positive weights and, for functions $g(y)$ of the type defined above, they satisfy the convergence property

$$
\lim _{m \rightarrow \infty} \sum_{j=1}^{m} v_{m, j} g\left(y_{m, j}\right)=\int_{a}^{b} g(y) d y ;
$$

furthermore, for the corresponding error term in (1.3) in general we have the estimates that we present next.

When in (1.3) $F(y)$ is of the form $\log \left|\frac{1-y}{1+y}\right| h(y)$, with $h(y)$ analytic, then the rules proposed in [14, Remark 1] and in [16] produce errors of the form $R_{m}(F)=O\left(m^{-l}\right)$ with $l>0$ that can be arbitrarily high. In particular we have

$$
R_{m}\left(\int_{-1}^{1} \frac{f(x, \cdot)}{x-\cdot} d x\right)=O\left(m^{-l}\right)
$$

whenever $f(x, y)$ is analytic with respect to both variables and, in the case of rule 14], the required smoothing exponent is sufficiently large. To see this it is sufficient to write

$$
F(y)=\int_{-1}^{1} \frac{f(x, y)}{x-y} d x=\int_{-1}^{1} \frac{f(x, y)-f(y, y)}{x-y} d x+f(y, y) \log \left|\frac{1-y}{1+y}\right|
$$

and recall the convergence estimates obtained in [14] (see also [5] Theorem 6]), [16].

This is the situation that most frequently occurs in BEM applications. However, if we want to consider the more general case of a function $f \in H_{p}(\mu, \mu)$ for some integer $p \geq 0$ and real $0<\mu \leq 1$, then the rule proposed in [14] appears more suitable. We recall that this rule originates from the combination of a smoothing change of variable $y=\gamma(s)$ of polynomial type (see (3.7),(3.8) below), mapping $[-1,1]$ onto $[-1,1]$ and with $\left|\gamma^{\prime}(s)\right| \leq c$ in the interval of integration, and the application of the $n$-point Gauss-Legendre rule to the transformed integral. By taking a proper smoothing exponent in $\gamma(s)$, for this rule we obtain the following convergence result. 
Theorem 5. When in (1.6) $f \in H_{p}(\mu, \mu)$, for some integer $p \geq 0$ and real $0<$ $\mu \leq 1$, then we have

$$
R_{m}\left(\int_{-1}^{1} \frac{f(x, \cdot)}{x-\cdot} d x\right)=O\left(m^{-p-\mu+\epsilon}\right)
$$

with $\epsilon>0$ arbitrarily small.

Proof. First we notice that we can always associate with the function

$$
g(y):=\int_{-1}^{1} \frac{f(x, y)-f(y, y)}{x-y} d x
$$

a sequence of polynomials $\left\{q_{k}(y) \in \Pi_{k}\right\}$ such that

$$
\left\|g-q_{k}\right\|_{\infty} \leq \frac{c}{k^{p+\mu-\epsilon}}
$$

with $\epsilon>0$ arbitrarily small.

To see this it is sufficient to recall the proofs given in 13. for results which in some sense are similar. Indeed, if $p_{j}(x, y) \in \Pi_{j}$ is a best (uniform) approximation polynomial defined by (2.6), i.e., such that $\left\|f-p_{j}\right\|_{\infty}=O\left(j^{-p-\mu+\epsilon}\right)$, then

$$
q_{2 j-1}(y):=\int_{-1}^{1} \frac{p_{j}(x, y)-p_{j}(y, y)}{x-y} d x
$$

is a polynomial of degree $2 j-1$, and furthermore (see [13, p.166])

$$
\left\|g-q_{2 j-1}\right\|_{\infty} \leq \frac{c}{j^{p+\mu-\epsilon}} .
$$

A polynomial $q_{2 j}(y)$ of degree $2 j$, satisfying the same estimate, can be easily obtained by adding to $q_{2 j-1}(y)$ a term of the type $y^{2 j} / j^{p+\mu}$.

Therefore, a sequence of polynomials $q_{k}(y)$, of degree $k=0,1,2, \ldots$, such that

$$
\left\|g-q_{k}\right\|_{\infty} \leq \frac{c}{k^{p+\mu-\epsilon}}
$$

can always be found, and

$$
\max _{-1 \leq s \leq 1}\left|\left[g(\gamma(s))-q_{k}(\gamma(s))\right] \gamma^{\prime}(s)\right| \leq \frac{c}{k^{p+\mu-\epsilon}} .
$$

To derive the final estimate we set in (1.3)

$$
F(y)=g(y)+g_{0}(y), \quad g_{0}(y):=f(y, y) \log \left|\frac{1-y}{1+y}\right|,
$$

hence write

$$
R_{m}(F)=R_{m}(g)+R_{m}\left(g_{0}\right) .
$$

By subtracting from $g$ a polynomial $q_{k}$ defined above, of proper degree $k$, and from $g_{0}(\gamma(s)) \gamma^{\prime}(s)$ a best (uniform) approximation polynomial, we obtain (3.2).

Remark 3. Incidentally we notice that (3.3) in particular implies

$$
E_{k}(g)_{\infty} \leq \frac{c}{k^{p+\mu-\epsilon}},
$$

hence, using the one-dimensional analogue of (2.5) (see [8, (2.1.2), (7.2.6)]), that

$$
\omega_{\phi}^{r}\left(g, \frac{1}{m}\right) \leq c m^{-r} \sum_{k=0}^{m}(k+1)^{r-1} E_{k}(g)_{\infty} \leq \frac{c}{m^{p+\mu-\epsilon}} .
$$


Therefore, for the global error (1.6) we have the bounds (see Theorem 3 and Remark 1)

$$
R_{m, n}(f)=O\left(n^{-l_{1}}\right)+O\left(m^{-l_{2}}\right),
$$

with $l_{i}>0$ that can be arbitrarily large, when $f$ is analytic and we use the rules proposed in [14], with a sufficiently large smoothing exponent $q$, or in [16]. Whenever $f \in H_{p}(\mu, \mu)$ and we use the quadrature suggested in [14] (with a sufficiently large smoothing exponent) we have

$$
R_{m, n}(f)=O\left(n^{-p-\mu} \log n\right)+O\left(m^{-p-\mu+\epsilon}\right),
$$

with $\epsilon>0$ arbitrarily small.

To check the validity of these bounds, we consider the following integral

$$
I_{1}(f)=\int_{0}^{1} \oint_{0}^{1} \frac{f(x, y)}{x-y} d x d y
$$

first with $f(x, y) \equiv f_{1}(x, y)=\log \left[(x+2)^{2}+y^{2}\right]$, and then with $f(x, y) \equiv f_{2}(x, y)=$ $|y-1.5 x|^{2.6}+(x-0.3)^{2}$, and apply to it formula (1.6) with $m=n$. Here we take $m=n$ only for simplicity, since our main goal is to test the theoretical convergence estimate we have derived. From the computational point of view there might be more convenient choices of $m$.

In particular, as rule (1.3) we take the $n$-point Gauss-Legendre rule combined with the smoothing procedure suggested in [14]. This procedure is given by the change of variable

$$
y=\gamma_{1}(s):=\frac{[(q-1) !]^{2}}{(2 q-1) !} \int_{0}^{s} x^{q-1}(1-x)^{q-1} d x, q>1
$$

It is a nondecreasing function which maps $[0,1]$ onto $[0,1]$ and has all derivatives of order $k, k=1, \ldots, q-1$, vanishing at the endpoints 0,1 . Recalling the convergence estimate derived in [5] Theorem 6], for the above procedure in the case of $I_{1}\left(f_{1}\right)$ we will have $R_{n}=O\left(n^{-2 q} \log n\right)$, hence $R_{n, n}(f)=O\left(n^{-2 q} \log n\right)$ since the function $f_{1}$ is analytic in the domain of integration.

In the case of $I_{1}\left(f_{2}\right)$ we have $f_{2} \in H_{2}(0.6,0.6)$. From Theorem 3 and Remark 1 the bound $\left|R_{n}^{I}\left(f_{2} ; y\right)\right| \leq c \bar{h}_{\alpha} n^{-2.6} \log n$ then follows; taking $q=2$ in (3.7), from (3.5) we obtain $R_{n, n}\left(f_{2}\right)=O\left(n^{-2.6+\epsilon}\right)$. Actually, in this case, a finer error estimate gives $O\left(n^{-2.6+\epsilon}\right)+O\left(n^{-2 q} \log n\right)$.

The corresponding relative errors are reported in the tables below. As exact values we consider the approximations given by our quadrature rule with $q=4$ and $n=128$ :

$$
I_{1}\left(f_{1}\right) \cong 0.312377389077288, \quad I_{1}\left(f_{2}\right) \cong 0.741458766596067 .
$$

The quantity $E O C=\log \frac{e_{n}}{e_{2 n}} / \log 2$, where $e_{k}$ is the absolute error produced by the rule with $n=m=k$, denotes the estimated order of convergence (see Tables 1 and 2).

Finally we consider the integral

$$
I_{2}\left(f_{1}\right)=\int_{-1}^{0} \int_{0}^{1} \frac{f_{1}(x, y)}{x-y} d x d y
$$

and apply the procedure suggested in [1]. Since in this case the associated function $F(y)$ (see (1.3)) has a log singularity only at the origin, it is sufficient to introduce 
TABLE 1. Relative errors and EOCs for $I_{1}\left(f_{1}\right)$.

\begin{tabular}{|c|c|c|c|c|c|c|c|c|c|c|}
\hline$n$ & $q=1$ & EOC & $q=2$ & EOC & $q=3$ & $\mathrm{EOC}$ & $q=4$ & EOC & $q=5$ & EOC \\
\hline$\overline{\overline{4}}$ & $\overline{99.6 \mathrm{E}-02}$ & & $\overline{2.2 \mathrm{E}-02}$ & & $\overline{2.5 \mathrm{E}-02}$ & & $\overline{22.1 \mathrm{E}-01}$ & & $\overline{5.1 \mathrm{E}-01}$ & \\
\hline & & 1.88 & & 3.67 & & 6.54 & & 11.9 & & 11.5 \\
\hline 0 & $2.6 \mathrm{E}-02$ & 1.93 & $1.7 \mathrm{E}-03$ & 3.86 & $2.7 \mathrm{E}-04$ & 5.93 & $5.6 \mathrm{E}-05$ & 7.59 & $1.8 \mathrm{E}-04$ & 12.5 \\
\hline 16 & $6.8 \mathrm{E}-03$ & & $1.2 \mathrm{E}-04$ & & $4.5 \mathrm{E}-06$ & & $2.9 \mathrm{E}-07$ & & $3.0 \mathrm{E}-08$ & \\
\hline 32 & $18 \mathrm{E}-03$ & 1.96 & $7.9 \mathrm{E}-06$ & 3.92 & $7.5 \mathrm{E}-08$ & 5.90 & $12 \mathrm{E}-09$ & .91 & $30 \mathrm{E}-11$ & 9.98 \\
\hline & & 1.98 & & 3.96 & & 5.94 & & 7.93 & & 9.05 \\
\hline 64 & $4.5 \mathrm{E}-04$ & & $5.1 \mathrm{E}-07$ & & $1.2 \mathrm{E}-09$ & & $4.9 \mathrm{E}-12$ & & $5.7 \mathrm{E}-14$ & \\
\hline 128 & $1.1 \mathrm{E}-04$ & 1.99 & $3.2 \mathrm{E}-08$ & 3.98 & & 5.97 & & & & \\
\hline
\end{tabular}

TABLE 2. Relative errors and EOCs for $I_{1}\left(f_{2}\right)$.

\begin{tabular}{|c|c|c|c|c|c|c|c|c|c|c|}
\hline$n$ & $q=1$ & EOC & $q=2$ & EOC & $q=3$ & $\mathrm{EOC}$ & $q=4$ & $\mathrm{EOC}$ & $q=5$ & $\mathrm{EOC}$ \\
\hline$\overline{4}$ & $\overline{2.7 \mathrm{E}-02}$ & & $\overline{2.5 \mathrm{E}-02}$ & & $\overline{1.9 \mathrm{E}-02}$ & & $4.4 \mathrm{E}-02$ & & $1.4 \mathrm{E}-01$ & \\
\hline & & 2.02 & & 4.75 & & 5.05 & & 7.07 & & 8.62 \\
\hline 8 & $0.0 \mathrm{E}-03$ & 1.91 & $9.2 \mathrm{E}-\mathrm{-U} 4$ & 2.79 & $5.8 \mathrm{E}$ & 3.45 & $3.3 \mathrm{E}-04$ & 2.61 & 3.7 & 2.08 \\
\hline 16 & $1.8 \mathrm{E}-03$ & & $1.3 \mathrm{E}-04$ & & $5.3 \mathrm{E}-05$ & & $5.3 \mathrm{E}-05$ & & $8.7 \mathrm{E}-05$ & \\
\hline 32 & $4.6 \mathrm{E}-04$ & 1.95 & $4.2 \mathrm{E}-06$ & & $6.9 \mathrm{E}-07$ & 6.24 & $4.8 \mathrm{E}-06$ & & $1.1 \mathrm{E}-06$ & 5.26 \\
\hline 64 & $1.2 \mathrm{E}-04$ & 1.98 & $1.6 \mathrm{E}-07$ & 4.71 & $1.8 \mathrm{E}-07$ & 1.93 & & 7.69 & & 1.09 \\
\hline 128 & $2.9 \mathrm{E}-05$ & 1.99 & & 2.94 & & 4.51 & & & & \\
\hline
\end{tabular}

the simpler change of variable

$$
y=\gamma_{2}(s):=s^{q}, q \geq 1,
$$

which leads to the bound $R_{n}\left(f_{1}\right)=O\left(n^{-2 q} \log n\right)$. For the evaluation of the inner integral we proceed as suggested in [1] (see also [7]): we use (1.2) when, for example, $-0.05=y_{0}<y<0$, and the $n$-point Gauss-Legendre formula otherwise. We recall that in this particular case the remainder term of the latter rule is $O\left(n^{-l}\right)$ with $l$ arbitrarily large; therefore it can also be bounded by (2.34). This allows us to obtain the final estimate

$$
R_{n, n}\left(f_{1}\right)=O\left(n^{-2 q} \log n\right) .
$$

The above choice of $y_{0}$ is purely indicative. We recall that if it is too large, then the recurrence relationships used to compute the weights of the internal quadrature rule are unstable (see [7]); if, on the contrary, it is too small, then the Gauss-Legendre rule that we use to compute the inner integral whenever $-1<y \leq y_{0}$ would require too many points to produce the required accuracy. A good choice of $y_{0}$ should be suggested by a criterion analogous to that used in [7].

Some numerical results are reported in Table 3 . In this example as reference value for the computation of the errors we have considered the approximation obtained with $q=5$ and $n=64$ :

$$
I_{2}\left(f_{1}\right) \cong 2.411514970798973 .
$$

All computations have been performed using double precision arithmetic (16 digits); nodes and weights of the Gauss-Legendre formulas have been computed to about 15 digit accuracy. 
TABLE 3. Relative errors and EOCs for $I_{2}\left(f_{1}\right)$.

\begin{tabular}{|c|c|c|c|c|c|c|c|c|c|c|}
\hline$n$ & $q=1$ & $\mathrm{EOC}$ & $q=2$ & $\mathrm{EOC}$ & $q=3$ & $\mathrm{EOC}$ & $q=4$ & $\mathrm{EOC}$ & $q=5$ & $\mathrm{EOC}$ \\
\hline$\overline{4}$ & $2.3 \mathrm{E}-02$ & & $4.1 \mathrm{E}-04$ & & $3.7 \mathrm{E}-04$ & & $2.9 \mathrm{E}-04$ & & $9.1 \mathrm{E}-04$ & \\
\hline & & 2.20 & & 3.77 & & 2.89 & & 6.31 & & 4.43 \\
\hline 8 & $5.1 \mathrm{E}-03$ & 1.92 & $3.0 \mathrm{E}-05$ & 1.96 & $5.0 \mathrm{E}-05$ & 9.18 & $3.7 \mathrm{E}-06$ & 12.1 & $4.2 \mathrm{E}-\mathrm{Ub}$ & 17.8 \\
\hline 16 & $1.3 \mathrm{E}-03$ & & $7.8 \mathrm{E}-06$ & & $8.6 \mathrm{E}-08$ & & $8.5 \mathrm{E}-10$ & & $1.8 \mathrm{E}-10$ & \\
\hline & & 1.96 & & 3.92 & & 5.89 & & 6.99 & & 11.9 \\
\hline 32 & $3.4 \mathrm{E}-04$ & & $5.2 \mathrm{E}-07$ & & $1.5 \mathrm{E}-09$ & & $6.7 \mathrm{E}-12$ & & $4.6 \mathrm{E}-14$ & \\
\hline & & 1.98 & & 3.96 & & 5.94 & & 7.84 & & \\
\hline 64 & 8.7E-05 & & $3.3 \mathrm{E}-08$ & & $2.4 \mathrm{E}-11$ & & $2.9 \mathrm{E}-14$ & & & \\
\hline 128 & $2.2 \mathrm{E}-05$ & 1.99 & 2.7E-09 & 3.02 & & & & & & \\
\hline
\end{tabular}

\section{REFERENCES}

[1] A.Aimi, M.Diligenti, G.Monegato, Numerical integration schemes for the BEM solution of hypersingular integral equations, Int. J. Numer. Meth. Engng. 45, 1999, pp.1807-1830. CMP 99:16

[2] R.L.Bisplinghoff, H.Ashley, Aeroelasticity, Addison-Wesley, Reading, Mass., 1962, pp.188293. MR 27:1022

[3] G.Criscuolo, G.Mastroianni, On the convergence of an interpolatory product rule for evaluating Cauchy principal value integrals, Math. Comp. 48, 1987, pp.725-735. MR 88m:65038

[4] G.Criscuolo, G.Mastroianni, On the uniform convergence of Gaussian quadrature rules for Cauchy principal value integrals, Numer. Math. 54, 1989, pp.445-461. [MR 90h:65023

[5] G.Criscuolo, G.Mastroianni, G.Monegato, Convergence properties of a class of product formulas for weakly singular integral equations, Math. Comp. 55, 1990, pp.213-230. MR 90m:65230

[6] G.Criscuolo, G.Mastroianni, Mean and uniform convergence of quadrature rules for evaluating the finite Hilbert transform, in: Progress in Approximation Theory (P.Nevai, A.Pinkus, eds.), Academic Press, Boston, 1991, pp.141-175. MR 92f:65035

[7] M.Diligenti, G.Monegato, Integral evaluation in the BEM solution of (hyper)singular integral equations. 2D problems on polygonal domains, J. Comput. Appl. Math.81, 1997, pp.29-57. MR 98k:65073

[8] Z.Ditzian, V.Totik, Moduli of Smoothness, Spinger-Verlag, Heidelberg, 1987. MR 89h:41002

[9] A.Erdely et al., Higher Transcendental Functions, Bateman Manuscript Project, vol. I, McGraw-Hill, New York, 1953.

[10] G.Mastroianni, M.G.Russo, Lagrange interpolation in some weighted uniform spaces, Facta Universitatis, ser. Math. Inform. 12, 1997, pp.185-201. MR 99m:41004

[11] G.Mastroianni, M.G.Russo, Lagrange interpolation in weighted Besov spaces, Constr. Approx. 15, 1999, pp.257-289. MR 2000b:41004

[12] G.Monegato, The numerical evaluation of one-dimensional Cauchy principal value integrals, Computing 29, 1982, pp.337-354. MR 84c:65044

[13] G.Monegato, Convergence of product formulas for the numerical evaluation of certain two-dimensional Cauchy principal value integrals, Numer. Math. 43, 1984, pp.161-173. MR 85h:65049

[14] G.Monegato, L.Scuderi, High order methods for weakly singular integral equations with non smooth input functions, Math. Comp. 67, 1998, pp.1493-1515. MR 99a:65192

[15] G.Monegato, J.Lyness, On the numerical evaluation of a particular singular two-dimensional integral, Math. Comp. 33, 1979, pp.993-1002. MR 80c:65050

[16] M.Mori, Quadrature formulas obtained by variable transformation and the DE-rule, J. Comput. Appl. Math. 12-13, 1985, pp.119-130. MR 86f:65051

[17] P.Nevai, Mean convergence of Lagrange interpolation I, J. Approx. Theory 18, 1976, pp.363377. MR 54:13375

[18] C.S.Song, Numerical integration of a double integral with a Cauchy-type singularity, AIAA J. 7, 1969, pp.1389-1390. MR 39:6516

[19] G.Szegö, Orthogonal Polynomials, Amer. Math. Soc., vol. 23, Providence, R.I., 1975. MR 51:8724 
Dipartimento di Matematica, Università della Basilicata, I-85100 Potenza, Italy

E-mail address: mg039sci@unibas.it

Dipartimento di Matematica, Politecnico di Torino, I-10129 Torino, Italy

E-mail address: Monegato@polito.it 